\title{
Antipsychotic-Like Effect of Trimetazidine in a Rodent Model
}

\author{
Oytun Erbaş, ${ }^{1}$ Hüseyin Serdar Akseki, ${ }^{2}$ Betuil Eliküiçük, ${ }^{3}$ and Dilek Taşkıran ${ }^{4}$ \\ ${ }^{1}$ Gaziosmanpaşa University School of Medicine, Department of Physiology, 60100 Tokat, Turkey \\ ${ }^{2}$ Tavsanl State Hospital, Department of Psychiatry, 43300 Kütahya, Turkey \\ ${ }^{3}$ Manisa Mental Health and Illnesses Hospital, 45000 Manisa, Turkey \\ ${ }^{4}$ Ege University School of Medicine, Department of Physiology, 35100 Izmir, Turkey \\ Correspondence should be addressed to Dilek Taşkıran; dilek.taskiran@ege.edu.tr
}

Received 16 August 2013; Accepted 6 September 2013

Academic Editors: N. Berretta, J. L. Labandeira-García, A. Takano, and Y. Yoshiyama

Copyright (C) 2013 Oytun Erbaş et al. This is an open access article distributed under the Creative Commons Attribution License, which permits unrestricted use, distribution, and reproduction in any medium, provided the original work is properly cited.

\begin{abstract}
Trimetazidine (TMZ) has been used as an anti-ischemic agent for angina pectoris, chorioretinal disturbances, and vertigo. Also, it can induce extrapyramidal type adverse reaction such as parkinsonism, gait disorder, and tremor via blockade of D2 receptors. In the present study, we evaluated the effect of TMZ on novelty-induced rearing behavior and apomorphine-induced stereotypy behavior in male rats. Four groups of rat $(n=7)$ were administrated with TMZ (10 and $20 \mathrm{mg} / \mathrm{kg}$, i.p.), chlorpromazine $(1 \mathrm{mg} / \mathrm{kg}$, i.p.), or isotonic saline. One hour later, apomorphine $(2 \mathrm{mg} / \mathrm{kg}$, s.c.) was administrated to each rat. Our results showed that both doses of TMZ significantly decreased the rearing behavior in rats, whereas the decrease with chlorpromazine was higher. TMZ also decreased the stereotypy scores in a dose-dependent manner. We concluded that TMZ has beneficial effects on rearing behavior and stereotypy, which are accepted to be indicators of antipsychotic effect. Taken together, with its antioxidative and cytoprotective properties, TMZ is worthy of being investigated for its anti-psychotic effects as a primary or an adjunctive drug.
\end{abstract}

\section{Introduction}

Schizophrenia is a psychiatric disorder, which is thought to have close relationship with hyperdopaminergic activity [1]. Based on pharmacological, as well as clinical, evidence, hyperdopaminergic activity in the mesolimbic pathway is believed to be responsible for the positive symptoms, whereas hypodopaminergic activity in the mesocortical pathway triggers cognitive impairment and affective symptoms [2, 3]. Drugs used in the treatment of the disease have the potential to cause extrapyramidal side effects by blocking D2 dopamine receptors mainly located in the striatum $[4,5]$.

Majority of the studies reported a significant imbalance between oxidative stress levels and antioxidative enzyme activities in schizophrenia. For example, Pazvantoglu et al. [6] demonstrated that the severity of the symptoms was negatively correlated with the total antioxidant potentials, whereas Padurariu et al. [7] found conflicting results demonstrating the increased superoxide dismutase (SOD) activity and decreased glutathione peroxidase (GPx) activity in patients with schizophrenia compared to controls. However, the studies on the lipid peroxidation markers, such as malonyl dialdehyde (MDA) and 4-hydroxynonenal (4-HNE), showed more consistent results [7-10]. Wang et al. showed increased levels of 4-HNE in schizophrenic patients compared to normal controls [8]. Also, MDA levels have been found elevated in peripheral tissues of schizophrenic patients [7, 9].

Trimetazidine (TMZ; 1-[2,3,4-trimethoxybenzyl]piperazine), an anti-ischemic drug, has been used in cardiology practice due to its protective effects particularly against myocardial ischemia and reperfusion injury [1114]. Trimetazidine is thought to carry out its effects via supporting cellular homeostasis during ischemia-reperfusion injury. Both in vivo and ex vivo trials have revealed that TMZ reduces intracellular acidosis, regulates $\mathrm{Na}^{+}$and $\mathrm{Ca}^{2+}$ levels, lessens membrane damage, preserves mitochondrial functions, regulates myocardial glucose utilization in favour of glucose use, and limits neutrophil infiltration in the ischemic area [15-19].

For decades, TMZ has been demonstrated to be safe and effective in patients with coronary artery disease and neurosensory ischaemia. However, recent studies have reported 
that it can also induce extrapyramidal type adverse reaction such as parkinsonism, gait disorder, and tremor [2022]. TMZ has the same piperazine core as cinnarizine and flunarizine, calcium channel blockers, which interacts with dopamine receptors (and particularly striatal dopamine D2 receptors) and leads to extrapyramidal disorders [20,23].

Despite many studies depicting its extrapyramidal side effects, which are thought to be in close relationship with D2 dopamine receptor blockade, to date there are no preclinical studies about the antipsychotic-like effect of TMZ. Hence, in the present study, we hypothesized that TMZ may produce an antipsychotic-like effect in a rodent model for psychosis. In order to determine its efficacy in psychosis, we compared the effects of TMZ and chlorpromazine, a conventional antipsychotic drug, by evaluating the novelty-induced rearing and apomorphine-induced stereotypic behavior in rats.

\section{Materials and Methods}

2.1. Animals and Housing Conditions. Twenty-eight adult male Sprague Dawley rats (220-240 g) were included in the study. All animals were kept under standard $12 \mathrm{~h}$ light/dark cycle in a temperature controlled $\left(22 \pm 2^{\circ} \mathrm{C}\right)$ environment with ad libitum access to rodent chow. All experimental procedures were performed during the light cycle (from 10:00 to 16:00). The experimental protocol performed in the study was approved by the Institutional Animal Care and Ethics Committee of the Ege University.

2.2. Drugs. All drugs were freshly prepared. Apomorphine hydrochloride (Sigma Chemical Co., St. Louis, MO) was dissolved in saline containing $0.1 \%$ ascorbic acid prior to experiments. TMZ (Servier Drug Company, Istanbul, Turkey) was dissolved in saline. Saline $(0.9 \% \mathrm{NaCl})$ was used as control solution. All solutions were administered intraperitoneally (i.p.) in a volume of $1 \mathrm{~mL} / \mathrm{kg}$ body weight.

2.3. Assessment of Novelty-Induced Rearing Behavior. Novelty-induced rearing behavior is used to assess the central excitatory locomotor behavior in rodents [24]. Four groups of rat $(n=7)$ were administered TMZ (10 and $20 \mathrm{mg} / \mathrm{kg}$, i.p.), chlorpromazine (1 mg/kg; i.p.), or isotonic $\mathrm{NaCl}(1 \mathrm{~mL} / \mathrm{kg}$, i.p.). One hour later, novelty-induced rearing behavior was explored by placing the animals directly from home cages to a transparent Plexiglas cage $(45 \mathrm{~cm} \times 25 \mathrm{~cm} \times 25 \mathrm{~cm})$. The rearing frequency (number of times the animal stood on its hind limbs or with its fore limbs against the walls of the observation box or free in the air) was recorded for $10 \mathrm{~min}$. All rats were monitored individually by two observers who were blinded to the study groups. The arena was cleaned with 5\% alcohol to eliminate olfactory bias before beginning a fresh animal.

\subsection{Apomorphine-Induced Stereotypic Behavior Test. Meso-} limbic and nigrostriatal dopaminergic pathways play crucial roles in the mediation of locomotor activity and stereotyped behavior. Apomorphine-induced stereotypy is due to the stimulation of dopamine receptors and has been used as a convenient method for in vivo screening of dopamine agonists or antagonists and assessment of dopaminergic activity $[25,26]$.

Briefly, four groups of rat $(n=7)$ were administered TMZ (10 and $20 \mathrm{mg} / \mathrm{kg}$, i.p.), chlorpromazine (1 mg/kg, i.p.), and isotonic saline $(1 \mathrm{~mL} / \mathrm{kg}$, i.p.). One hour later, apomorphine ( $2 \mathrm{mg} / \mathrm{kg}$, s.c.) was administered to each rat. First, rats were placed into the cylindrical metal cages $(18 \times 19 \mathrm{~cm})$ containing vertical (1 cm apart) and horizontal $(4.5 \mathrm{~cm}$ apart) metal bars $(2 \mathrm{~mm})$ with upper lid for 10 minutes for orientation period. After apomorphine administration, the rats were immediately placed back into the metal cages and observed for stereotypic behavior. Signs of stereotypy, which include mainly sniffing and gnawing, were observed and scored as follows: absence of stereotypy (0), occasional sniffing (1), occasional sniffing with occasional gnawing (2), frequent gnawing (3), intense continuous gnawing (4), and intense gnawing and staying on the same spot (5). The stereotypic behavior was rated after each minute, and mean of $15 \mathrm{~min}$ period was calculated and recorded [27].

2.5. Statistical Analysis. Statistical evaluation was performed by one-way analysis of variance (ANOVA). Post hoc Bonferroni test was used to identify differences between the experimental groups. Results are presented as mean \pm SEM. A value of $P<0.05$ was considered to be significant.

\section{Results}

3.1. The Effect of TMZ on Novelty-Induced Rearing Behavior. Figure 1 represents the effects of TMZ and chlorpromazine treatment on rearing behavior. ANOVA results revealed significant differences between the groups $(P<0.0005)$. Post-hoc Bonferroni test demonstrated a highly significant reduction in rearing behavior in TMZ (10 and $20 \mathrm{mg} / \mathrm{kg}$ ) and chlorpromazine $(1 \mathrm{mg} / \mathrm{kg})$ administered rats compared to saline group ( $P=0.037, P=0.000, P=0.000$, resp.). The inhibitory effect of TMZ on rearing behavior was dose dependent, being more evident at a higher dose $(20 \mathrm{mg} / \mathrm{kg})$.

\subsection{The Effect of TMZ on Apomorphine-Induced Stereotypic} Behavior Test. Figure 2 depicts the effects of TMZ and chlorpromazine treatment on stereotypy scores. ANOVA results showed significant differences between the groups $(P<$ 0.0005). Post-hoc Bonferonni test demonstrated a highly significant decrease in stereotypy scores in both doses of TMZ and chlorpromazine compared to saline group $(P=0.004$, $P=0.000, P=0.000$, resp.). The decrease was significantly greater with $20 \mathrm{mg} / \mathrm{kg}$ of TMZ compared to $10 \mathrm{mg} / \mathrm{kg}(P=$ $0.033)$.

\section{Discussion}

This study demonstrates the beneficial effects of TMZ on rearing behavior and stereotypy, which are accepted to be indicators of anti-psychotic effect. Theoretically, antipsychotic effect is mediated by means of antidopaminergic activity in certain regions of central nervous system. But 


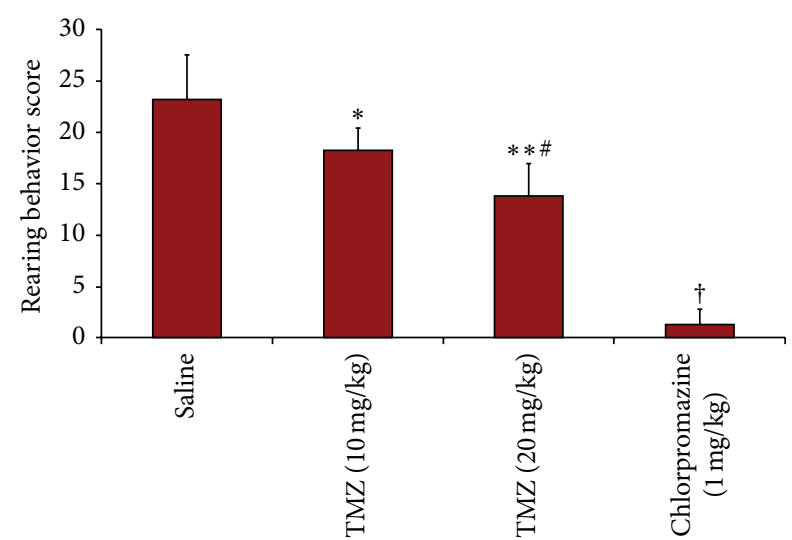

FIGURE 1: Rearing behavior scores. Data are expressed as mean \pm SEM. Statistical analysis was performed by one-way analysis of variance (ANOVA) and Bonferroni's post hoc test. * different from saline, $P=0.037,{ }^{* *}$ different from saline, $P=0.000,{ }^{*}$ different from TMZ $(10 \mathrm{mg} / \mathrm{kg}), P=0.029,{ }^{\dagger}$ different from other groups, $P=0.000$.

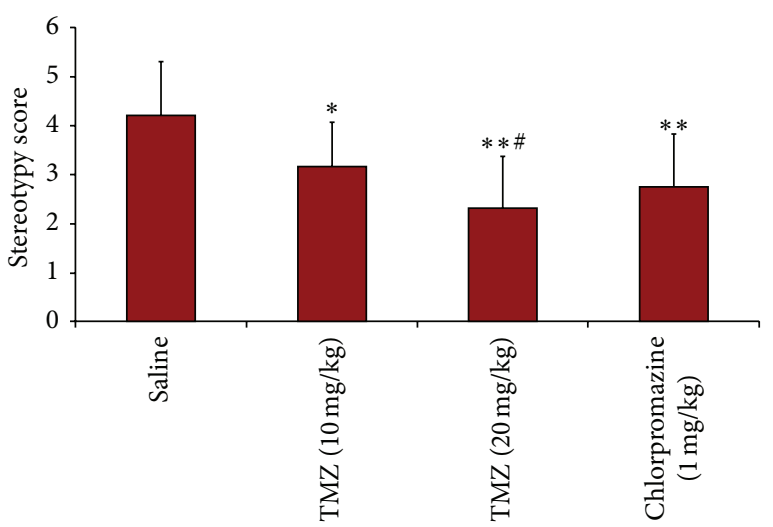

FIGURE 2: Apomorphine-induced stereotypy scores. Data are expressed as mean \pm SEM. Statistical analysis was performed by oneway analysis of variance (ANOVA) and Bonferroni's post hoc test. ${ }^{*}$ different from saline, $P=0.004,{ }^{* *}$ different from saline, $P=0.000$, \# different from TMZ $(10 \mathrm{mg} / \mathrm{kg}), P=0.033$.

adverse drug effects have brought a big burden for the patients. Therefore, clinical and nonclinical investigations focused on new drugs, which cause fewer side effects.

Exposure of rodents to a new environment causes novelty-induced behavior syndrome consisting of rearing, grooming, and wet-dog shakes. The novelty-induced rearing behavior response is regulated by various neurotransmitter systems including $\mathrm{GABA}_{\mathrm{A}}$, opioid, and dopamine D2 receptors [28]. In a previous study, Tejashree et al. examined the anti-psychotic-like effects of liraglutide, a GLP1 agonist, and sitagliptin, which is a dipeptidyl peptidase (4DPP-4) inhibitor [29]. Liraglutide and sitagliptin are US FDA approved medications for the treatment of type 2 diabetes mellitus. Liraglutide treatment significantly attenuated apomorphine-induced cage climbing behavior, which is thought to be first preclinical evidence for anti-psychotic-like effect [29]. As TMZ reduces the utilization of fatty acids in favour of carbohydrates, it may share a common mechanism with liraglutide in the cellular level. In Tejashree's study, liraglutide showed equal effect with haloperidol in reversing apomorphine-induced cage climbing behavior. In the present study, TMZ decreased rearing behavior in rats in a doserelated manner, which is also reduced by chlorpromazine more efficiently. Chlorpromazine, a very effective antagonist of D2 dopamine receptors, exerts additional antiadrenergic, anticholinergic, and antihistaminergic effects [30]. Hence, the efficacy of chlorpromazine on rearing behavior may be associated with its sedative effect, which is mainly maintained by anticholinergic and antihistaminergic properties of that drug.

Sotoing Taïwe et al. [28] examined the effect of aqueous extract and alkaloid fraction of Crassocephalum bauchiense in rodents. Both aqueous extract and the alkaloid fraction caused dose-dependent inhibition in the rearing behavior, which is mediated through GABA-A, opioid, and D2 dopamine receptors [28]. Stereotypical behavior is a common feature manifested in schizophrenia and is increased by apomorphine probably through D2 receptors. Besides rearing behavior, Sotoing Taïwe et al. showed that aqueous extract and alkaloid fraction of Crassocephalum bauchiense decreased the apomorphine-induced stereotypy scores [28]. In our study, TMZ also significantly lessened the stereotypy scores.

Recently, Masmoudi et al. [20] reported the series of 21 cases that had extrapyramidal disorders associated with TMZ use. The TMZ-associated drug reactions were typical parkinsonism, gait disorders, and restless leg syndrome. The discontinuation of the drug led to total disappearance of symptoms in 16 patients [20]. Similarly, Bondon-Guitton et al. [21] reviewed drug-induced or drug-worsened parkinsonism cases reported to a pharmacovigilance center between 1993 and 2009 and reported three notifications with TMZ. Since TMZ owns a piperazine core in the chemical formula as in flunarizine, it can blockade D2 dopamine receptors and lead to extrapyramidal type adverse reaction [20,22].

In recent years, glutamate has been paid much attention because of its role in schizophrenia. Glutamatergic dysfunction is thought to be one of the possible etiologic factors in schizophrenia. It is known that glutamatergic transmission is primarily mediated through its metabolic and ionotropic receptors. The ionotropic receptors are $\mathrm{N}$-methylD-aspartate (NMDA), alpha-amino-3-hydroxy-5-methyl-4isoxazolepropionic acid (AMPA), and kainate receptors. Hypofunctional NMDA receptors are accused of being responsible in the pathogenesis of schizophrenia [30-32]. In a postmortem study, Noga et al. [33] reported increased AMPA binding in striatal structures such as caudate, putamen, and accumbens in schizophrenia using $\left[{ }^{3} \mathrm{H}\right] \mathrm{CNQX}$. Similarly, Zavitsanou et al. [34] found increased $\left[{ }^{3} \mathrm{H}\right] \mathrm{AMPA}$ binding in the superficial layers of cortex which suggests a postsynaptic compensation for impaired glutamatergic neurotransmission in schizophrenia. On the other hand, Dayanithi et al. [35] investigated the effects of TMZ on AMPA and kainate receptors in rat vestibular ganglion neurons, and they found that TMZ could be a potent antagonist of AMPA and 
kainate receptors. This study suggests that TMZ may have modulatory effects on non-NMDA glutamatergic receptors, which are thought to play an important role in schizophrenia.

It is well known that anti-psychotic drugs are the first choices for the treatment of schizophrenia. Majority of the studies indicated the increased levels of oxidative stress parameters after the treatment with classical antipsychotics. For example, Sagara et al. [36] showed that haloperidol induced a sixfold increase in levels of reactive oxygen species (ROS) and treatment of antioxidants, such as vitamin E, lowered the levels of ROS, and protected the cells. Similarly, Reinke et al. [37] revealed that haloperidol and clozapine were related with oxidative stress in the rat brain, but haloperidol-receiving group showed a higher increase compared to clozapine. More recently, Kropp et al. [38] measured the MDA levels in schizophrenic patients during treatment with first- and second-generation antipsychotics. According to their results, MDA levels in patients receiving clozapine, quetiapine, and risperidone were lower than the first-generation antipsychotic receiving group. They found that atypical anti-psychotics attenuated the oxidative stress and decreased oxidative damage markers. On the other hand, it has been claimed that increased oxidative stress seen in some clozapine treated patients could be related to the illness severity since clozapine is mainly used in refractory patients [39].

All these studies point out that there is a growing body of evidence proving the importance of oxidative stress in schizophrenia both in pathogenesis and treatment modalities. So, it is suggested that antioxidants might be useful in the treatment of schizophrenia. For instance, Dakhale et al. [40] indicated that vitamin C and oral anti-psychotic combination reduced brief psychiatric rating scale scores and MDA levels. Zhang et al. showed that Ginkgo biloba extract (a powerful antioxidant) and haloperidol combination resulted in better positive and negative syndrome scale (PANSS) scores and reduced extrapyramidal side effects $[41,42]$. In a meta-analysis, Singh et al. [43] reported that Ginkgo biloba combined with anti-psychotics exhibited improvement in psychotic symptoms. As the majority of the studies show the improving effects of anti-oxidants as an adjunct therapy, we propose that TMZ might be beneficial with its obvious antioxidant effects in schizophrenia.

\section{Conclusion}

Considering the effects of TMZ on rearing behavior and stereotypical behavior in rats, we propose that TMZ may have anti-psychotic-like potential because of its antidopaminergic effects. On the other hand, TMZ, as an anti-oxidant and cytoprotective agent, can be useful in neuroprotection especially on early stages of psychosis or prepsychotic patients with insignificant symptoms. In addition, as glutamatergic excitotoxicity is responsible for the neurodegeneration and neuron loss, which is possibly related with negative symptoms and cognitive dysfunction, TMZ might be having a potential as a regulator on glutamatergic system. However, as we have little data about trimetazidine's anti-dopaminergic effects and glutamatergic modulating roles compared to its well-known anti-oxidant effects in psychotic patients, this study needs to be supported by further experimental and clinical research.

\section{Conflict of Interests}

The authors declare that they have no financial or commercial interests. No conflict of interests has been declared.

\section{References}

[1] P. Seeman, "Dopamine receptors and the dopamine hypothesis of schizophrenia," Synapse, vol. 1, no. 2, pp. 133-152, 1987.

[2] R. Kuepper, M. Skinbjerg, and A. Abi-Dargham, "The dopamine dysfunction in schizophrenia revisited: new insights into topography and course," Handbook of Experimental of Pharmacology, vol. 212, pp. 1-26, 2012.

[3] A. Abi-Dargham and H. Moore, "Prefrontal DA transmission at D1 receptors and the pathology of schizophrenia," Neuroscientist, vol. 9, no. 5, pp. 404-416, 2003.

[4] P. Seeman, "Atypical antipsychotics: mechanism of action," Canadian Journal of Psychiatry, vol. 47, no. 1, pp. 27-38, 2002.

[5] S. Kapur and P. Seeman, "Does fast dissociation from the dopamine $\mathrm{D}_{2}$ receptor explain the action of atypical antipsychotics? A new hypothesis," American Journal of Psychiatry, vol. 158, no. 3, pp. 360-369, 2001.

[6] O. Pazvantoglu, S. Selek, I. T. Okay et al., "Oxidative mechanisms in schizophrenia and their relationship with illness subtype and symptom profile," Psychiatry and Clinical Neurosciences, vol. 63, no. 5, pp. 693-700, 2009.

[7] M. Padurariu, A. Ciobica, I. Dobrin, and C. Stefanescu, "Evaluation of antioxidant enzymes activities and lipid peroxidation in schizophrenic patients treated with typical and atypical antipsychotics," Neuroscience Letters, vol. 479, no. 3, pp. 317-320, 2010.

[8] J.-F. Wang, L. Shao, X. Sun, and L. T. Young, "Increased oxidative stress in the anterior cingulate cortex of subjects with bipolar disorder and schizophrenia," Bipolar Disorders, vol. 11, no. 5, pp. 523-529, 2009.

[9] M. Kunz, C. S. Gama, A. C. Andreazza et al., "Elevated serum superoxide dismutase and thiobarbituric acid reactive substances in different phases of bipolar disorder and in schizophrenia," Progress in Neuro-Psychopharmacology and Biological Psychiatry, vol. 32, no. 7, pp. 1677-1681, 2008.

[10] S. J. Wood, M. Yücel, C. Pantelis, and M. Berk, "Neurobiology of schizophrenia spectrum disorders: the role of oxidative stress," Annals of the Academy of Medicine Singapore, vol. 38, no. 5, pp. 396-401, 2009.

[11] F. R. Boucher, D. J. Hearse, and L. H. Opie, "Effects of trimetazidine on ischemic contracture in isolated perfused rat hearts," Journal of Cardiovascular Pharmacology, vol. 24, no. 1, pp. 45-49, 1994.

[12] M. Cera, A. Salerno, G. Fragasso et al., "Beneficial electrophysiological effects of trimetazidine in patients with postischemic chronic heart failure," Journal of Cardiovascular Pharmacology and Therapeutics, vol. 15, no. 1, pp. 24-30, 2010.

[13] M. I. M. Noble, P. R. Belcher, and A. J. Drake-Holland, "Limitation of infarct size by trimetazidine in the rabbit," American Journal of Cardiology, vol. 76, no. 6, pp. 41B-44B, 1995.

[14] L. Dehina, F. Vaillant, A. Tabib et al., "Trimetazidine demonstrated cardioprotective effects through mitochondrial pathway 
in a model of acute coronary ischemia," Naunyn-Schmiedeberg's Archives of Pharmacology, vol. 386, no. 3, pp. 205-215, 2013.

[15] F. Reymond, G. Steyaert, P.-A. Carrupt et al., “The pH-partition profile of the anti-ischemic drug trimetazidine may explain its reduction of intracellular acidosis," Pharmaceutical Research, vol. 16, no. 5, pp. 616-624, 1999.

[16] J. F. Renaud, "Internal $\mathrm{pH}, \mathrm{Na}^{+}$, and $\mathrm{Ca}^{2+}$ regulation by trimetazidine during cardiac cell acidosis," Cardiovascular Drugs and Therapy, vol. 1, no. 6, pp. 677-686, 1988.

[17] C. Guarnieri, C. Finelli, M. Zini, and C. Muscari, "Effects of trimetazidine on the calcium transport and oxidative phosphorylation of isolated rat heart mitochondria," Basic Research in Cardiology, vol. 92, no. 2, pp. 90-95, 1997.

[18] V. Maupoil, L. Rochette, A. Tabard, P. Clauser, and C. Harpey, "Direct measurement of free radical generation in isolated rat heart by electron paramagnetic resonance spectroscopy: effect of trimetazidine," Advances in Experimental Medicine and Biology, vol. 264, pp. 373-376, 1990.

[19] F. M. Williams, K. Tanda, M. Kus, and T. J. Williams, "Trimetazidine inhibits neutrophil accumulation after myocardial ischaemia and reperfusion in rabbits," Journal of Cardiovascular Pharmacology, vol. 22, no. 6, pp. 828-833, 1993.

[20] K. Masmoudi, H. Masson, V. Gras, and M. Andréjak, "Extrapyramidal adverse drug reactions associated with trimetazidine: a series of 21 cases," Fundamental and Clinical Pharmacology, vol. 26, no. 2, pp. 198-203, 2012.

[21] E. Bondon-Guitton, S. Perez-Lloret, H. Bagheri, C. Brefel, O. Rascol, and J.-L. Montastruc, "Drug-induced parkinsonism: a review of 17 years' experience in a regional pharmacovigilance center in France," Movement Disorders, vol. 26, no. 12, pp. 22262231, 2011.

[22] J.-F. M. Massó, I. Martí, N. Carrera, J.-J. Poza, and A. L. de Munain, "Trimetazidine induces parkinsonism, gait disorders and tremor," Therapie, vol. 60, no. 4, pp. 419-422, 2005.

[23] T. Brücke, C. Wöber, I. Podreka et al., "D2 receptor blockade by flunarizine and cinnarizine explains extrapyramidal side effects. A SPECT study," Journal of Cerebral Blood Flow and Metabolism, vol. 15, no. 3, pp. 513-518, 1995.

[24] A. A. Ajayi and O. E. Ukponmwan, "Possible evidence of angiotensin II and endogenous opioid modulation of noveltyinduced rearing in the rat," African Journal of Medicine and Medical Sciences, vol. 23, no. 3, pp. 287-290, 1994.

[25] C. Reavill, A. Kettle, V. Holland, G. Riley, and T. P. Blackburn, "Attenuation of haloperidol-induced catalepsy by a $5-\mathrm{HT}_{2 \mathrm{C}}$ receptor antagonist," British Journal of Pharmacology, vol. 126, no. 3, pp. 572-574, 1999.

[26] S. M. Dong, Y. G. Kim, J. Heo, M. K. Ji, J. W. Cho, and B. S. Kwak, "YKP1447, a novel potential atypical antipsychotic agent," Korean Journal of Physiology and Pharmacology, vol. 13, no. 2, pp. 71-78, 2009.

[27] S. Amos, J. Abbah, B. Chindo et al., "Neuropharmacological effects of the aqueous extract of Nauclea latifolia root bark in rats and mice," Journal of Ethnopharmacology, vol. 97, no. 1, pp. 53-57, 2005.

[28] G. Sotoing Taïwe, E. N. Bum et al., "Anti-psychotic and sedative effects of the leaf extract of Crassocephalum bauchiense (Hutch) Milne-Redh (Asteracea) in rodents," Journal of Ethnopharmacology, vol. 143, no. 1, pp. 213-220, 2012.

[29] S. D. Tejashree, N. S. Ajaykumar, B. L. James, and M. E. Khalid, "Anti-psychotic-like effect of GLP-1 agonist liraglutide but not DPP-4 inhibitor sitagliptin in mouse model for psychosis," Physiology and Behavior, vol. 114-115, pp. 38-41, 2013.
[30] S. M. Stahl, Stahl's Essential Psychopharmacology: Neuroscientific Basis and Practical Applications, Cambridge University Press, 2nd edition, 2000.

[31] J. A. Lieberman, "Is schizophrenia a neurodegenerative disorder? A clinical and neurobiological perspective," Biological Psychiatry, vol. 46, no. 6, pp. 729-739, 1999.

[32] A. H. C. Wong and H. H. M. van Tol, "Schizophrenia: from phenomenology to neurobiology," Neuroscience and Biobehavioral Reviews, vol. 27, no. 3, pp. 269-306, 2003.

[33] J. T. Noga, T. M. Hyde, M. M. Herman et al., "Glutamate receptors in the postmortem striatum of schizophrenic, suicide, and control brains," Synapse, vol. 27, pp. 168-176, 1997.

[34] K. Zavitsanou, P. B. Ward, and X.-F. Huang, "Selective alterations in ionotropic glutamate receptors in the anterior cingulate cortex in schizophrenia," Neuropsychopharmacology, vol. 27, no. 5, pp. 826-833, 2002.

[35] G. Dayanithi, G. Desmadryl, C. Travo, C. Chabbert, and A. Sans, "Trimetazidine modulates AMPA/kainate receptors in rat vestibular ganglion neurons," European Journal of Pharmacology, vol. 574, no. 1, pp. 8-14, 2007.

[36] Y. Sagara, "Induction of reactive oxygen species in neurons haloperidol," Journal of Neurochemistry, vol. 71, no. 3, pp. 10021012, 1998.

[37] A. Reinke, M. R. Martins, M. S. Lima, J. C. Moreira, F. DalPizzol, and J. Quevedo, "Haloperidol and clozapine, but not olanzapine, induces oxidative stress in rat brain," Neuroscience Letters, vol. 372, no. 1-2, pp. 157-160, 2004.

[38] S. Kropp, V. Kern, K. Lange et al., "Oxidative stress during treatment with first- and second-generation antipsychotics," Journal of Neuropsychiatry and Clinical Neurosciences, vol. 17, no. 2, pp. 227-231, 2005.

[39] C. S. Gama, M. Salvador, A. C. Andreazza, F. Kapczinski, and P. S. Belmonte-de-Abreu, "Elevated serum superoxide dismutase and thiobarbituric acid reactive substances in schizophrenia: a study of patients treated with haloperidol or clozapine," Progress in Neuro-Psychopharmacology and Biological Psychiatry, vol. 30, no. 3, pp. 512-515, 2006.

[40] G. N. Dakhale, S. D. Khanzode, S. S. Khanzode, and A. Saoji, "Supplementation of vitamin C with atypical antipsychotics reduces oxidative stress and improves the outcome of schizophrenia," Psychopharmacology, vol. 182, no. 4, pp. 494-498, 2005.

[41] X. Y. Zhang, D. F. Zhou, J. M. Su, and P. Y. Zhang, “The effect of extract of ginkgo biloba added to haloperidol on superoxide dismutase in patients with chronic schizophrenia," Journal of Clinical Psychopharmacology, vol. 21, no. 1, pp. 85-88, 2001.

[42] X. Y. Zhang, D. F. Zhou, P. Y. Zhang, G. Y. Wu, J. M. Su, and L. Y. Cao, "A double-blind, placebo-controlled trial of extract of ginkgo biloba added to haloperidol in treatment-resistant patients with schizophrenia," Journal of Clinical Psychiatry, vol. 62, no. 11, pp. 878-883, 2001.

[43] V. Singh, S. P. Singh, and K. Chan, "Review and meta-analysis of usage of ginkgo as an adjunct therapy in chronic schizophrenia," International Journal of Neuropsychopharmacology, vol. 13, no. 2, pp. 257-271, 2010. 

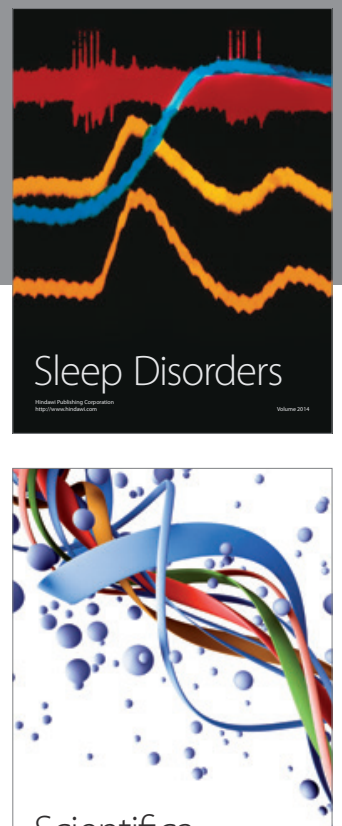

Scientifica
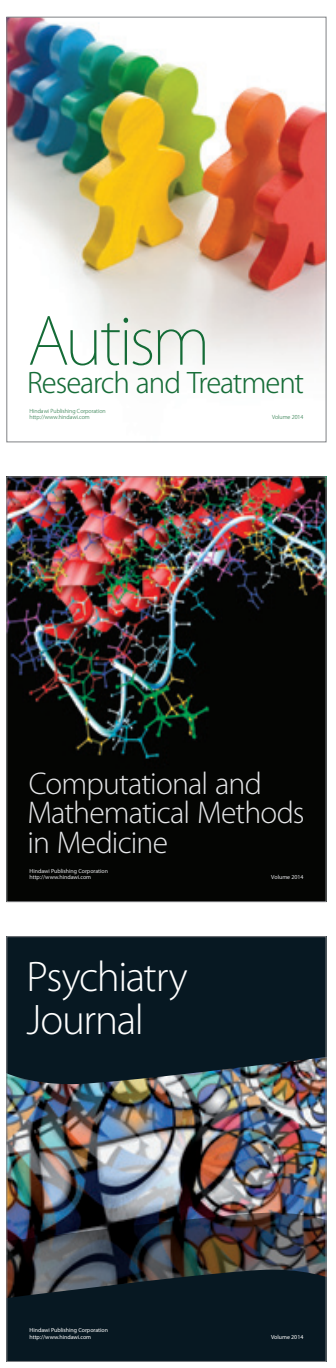
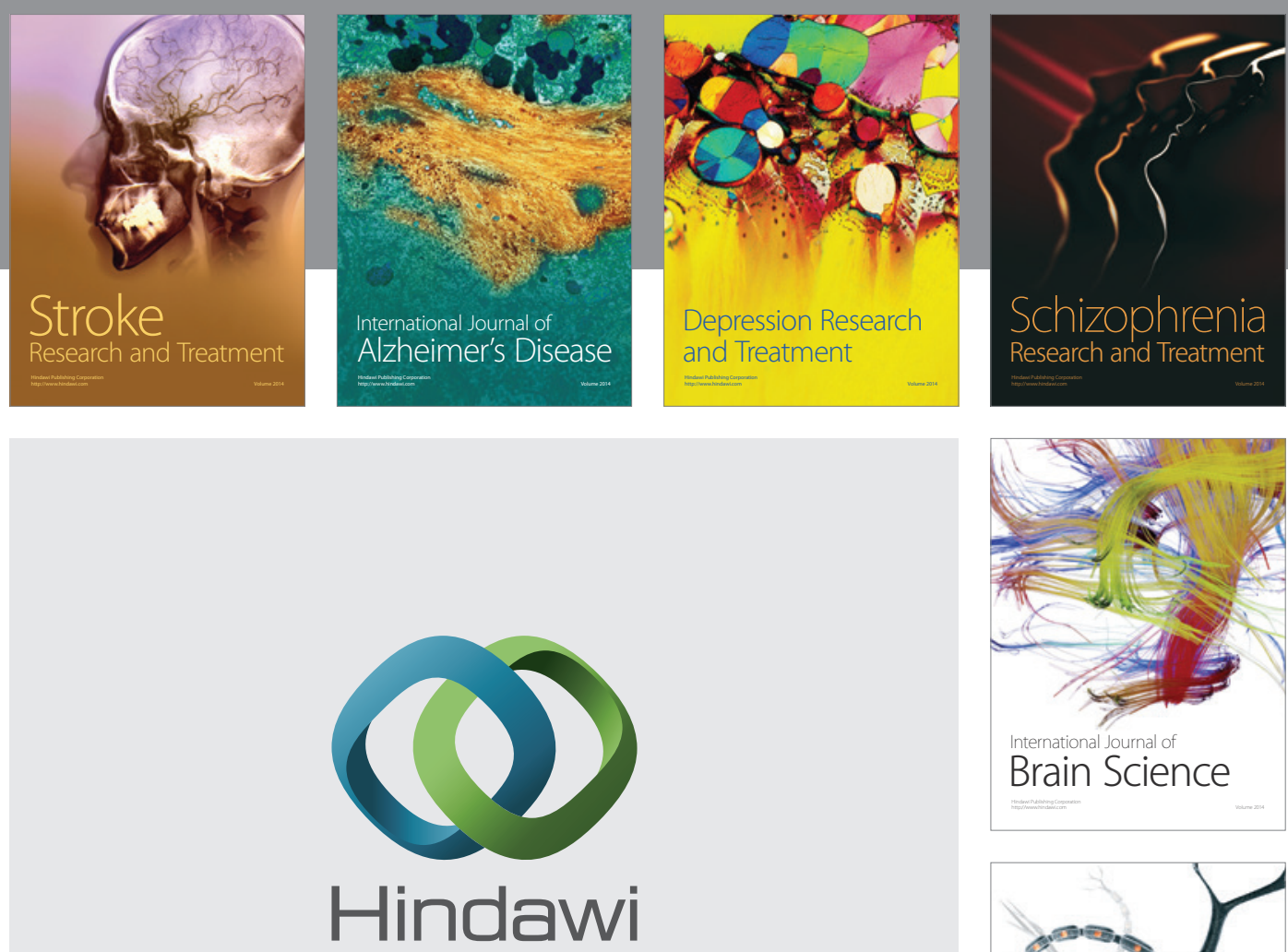

Submit your manuscripts at

http://www.hindawi.com
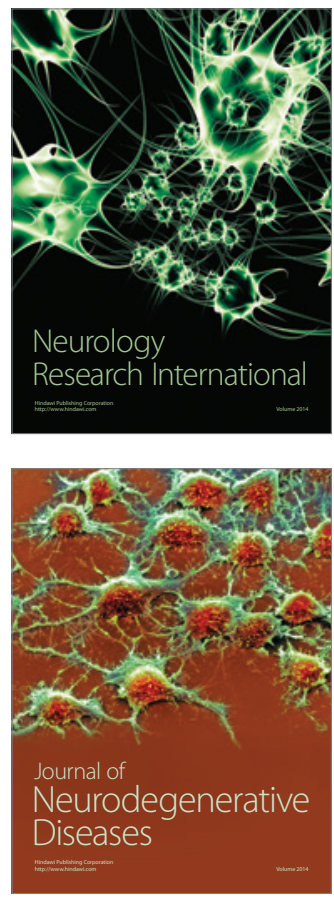

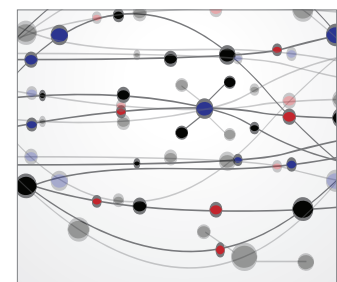

The Scientific World Journal
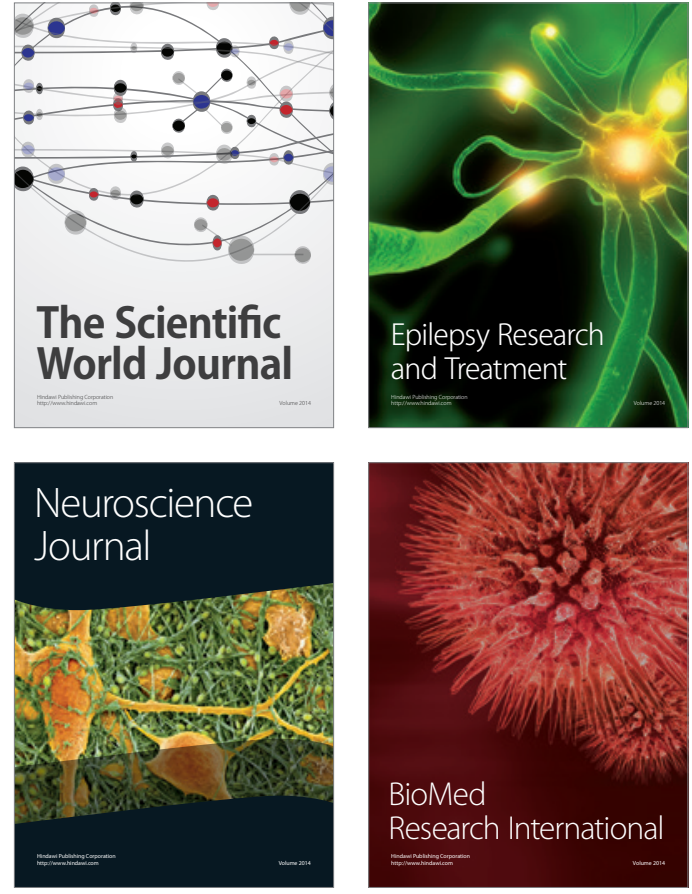

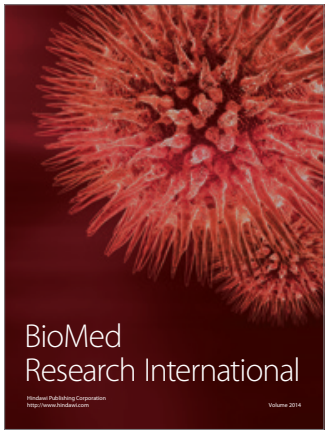

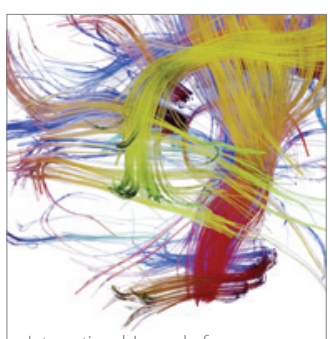

Brain Science

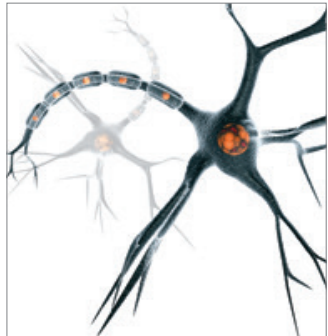

Neural Plasticity
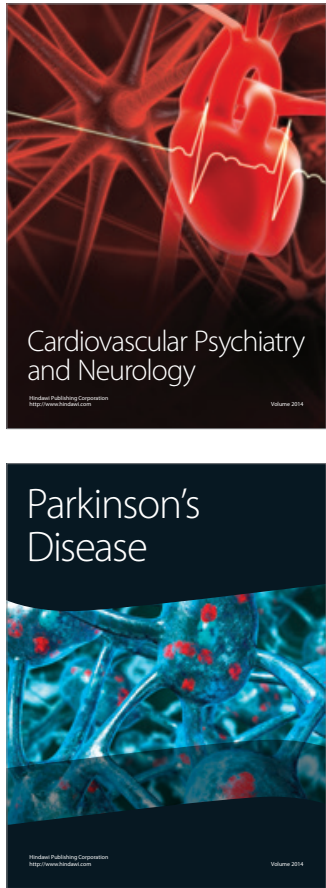